\title{
Correction to: The coming together of allosteric and phosphorylation mechanisms in the molecular integration of A2A heteroreceptor complexes in the dorsal and ventral striatal-pallidal GABA neurons
}

\author{
Dasiel O. Borroto-Escuela ${ }^{1,4} \cdot$ Luca Ferraro $^{2} \cdot$ Sarah Beggiato ${ }^{3} \cdot$ Manuel Narváez $^{4} \cdot$ Ramon Fores-Pons $^{4}$. \\ Jose E. Alvarez-Contino ${ }^{4,5} \cdot$ Karolina Wydra $^{6} \cdot$ Małgorzata Frankowska $^{6} \cdot$ Michael Bader $^{7} \cdot$ Małgorzata Filip $^{6}$. \\ Kjell Fuxe ${ }^{1}$
}

Published online: 17 September 2021

๑) Maj Institute of Pharmacology Polish Academy of Sciences 2021

\section{Correction to: Pharmacological Reports (2021) 73:1096-1108 https://doi.org/10.1007/s43440-021-00314-3}

In this article the e-mail addresses and the affiliation details for some of co-authors were incorrectly assigned. The correct authors information is as below.

Dasiel O. Borroto-Escuela ${ }^{1,4} \cdot$ Luca Ferraro ${ }^{2} \cdot$ Sarah Beggiato $^{3} \cdot$ Manuel Narváez ${ }^{4} \cdot$ Ramon Fores-Pons ${ }^{4} \cdot$ Jose E. Alvarez-Contino ${ }^{4,5}$. Karolina Wydra ${ }^{6}$. Małgorzata Frankowska $^{6} \cdot$ Michael Bader ${ }^{7} \cdot$ Małgorzata Filip $^{6} \cdot$ Kjell Fuxe $^{1}$

The original article can be found online at https://doi.org/10.1007/ s43440-021-00314-3.

Dasiel O. Borroto-Escuela

dasiel.borroto.escuela@ki.se

Kjell Fuxe

kjell.fuxe@ki.se

Luca Ferraro

frl@unife.it

Sarah Beggiato

sara.beggiato@unich.it

Manuel Narváez

mnarvaez@uma.es

Ramon Fores-Pons rforespons@gmail.com

Jose E. Alvarez-Contino erickjose@infomed.sld.cu

Karolina Wydra wydra@if-pan.krakow.pl

Małgorzata Frankowska frankow@if-pan.krakow.pl

Michael Bader mbader@mdc-berlin.de
Publisher's Note Springer Nature remains neutral with regard to jurisdictional claims in published maps and institutional affiliations.
Małgorzata Filip

mal.fl@if-pan.krakow.pl

1 Department of Neuroscience, Karolinska Institutet, Biomedicum (B0851), Solnavagen 9, 17177 Stockholm, Sweden

2 Department of Life Sciences and Biotechnology, University of Ferrara, Ferrara, Italy

3 Department of Medical, Oral and Biotechnological Sciences, University of Chieti-Pescara, 66100 Chieti, Italy

4 Facultad de Medicina, Instituto de Investigación Biomédica de Málaga, Universidad de Málaga, Campus de Teatinos s/n, 29071 Málaga, Spain

5 Policlínico Universitario Juan Bruno Zayas, Cifuentes, Villa Clara, Cuba

6 Department of Drug Addiction Pharmacology, Maj Institute of Pharmacology, Polish Academy of Sciences, 12 Smętna Street, 31343 Kraków, Poland

7 Max-Delbrück-Centrum Für Molekulare Medizin (MDC), Robert-Rössle-Strasse 10, 13125 Berlin, Germany 